\title{
DIFFERENCES OF COMPOSITION OPERATORS ON THE BLOCH SPACE IN THE POLYDISC
}

\author{
ZHONG-SHAN FANG and ZE-HUA ZHOU ${ }^{凶}$
}

\author{
(Received 18 August 2008)
}

\begin{abstract}
Let $\varphi$ and $\psi$ be holomorphic self-maps of the unit polydisc $U^{n}$ in the $n$-dimensional complex space, and denote by $C_{\varphi}$ and $C_{\psi}$ the induced composition operators. This paper gives some simple estimates of the essential norm for the difference of composition operators $C_{\varphi}-C_{\psi}$ from Bloch space to bounded holomorphic function space in the unit polydisc. The compactness of the difference is also characterized.
\end{abstract}

2000 Mathematics subject classification: primary 47B38; secondary 26A16, 32A16, 32A26, 32A30, 32A 37, 32A 38, 32H02, 47B33.

Keywords and phrases: essential norm, compact difference, composition operator, Bloch space, polydiscs.

\section{Introduction}

The algebra of all holomorphic functions with domain $\Omega$ will be denoted by $H(\Omega)$, where $\Omega$ is a bounded domain in $\mathbb{C}^{n}$. Let $\varphi=\left(\varphi_{1}(z), \ldots, \varphi_{n}(z)\right)$ and $\psi(z)=$ $\left(\psi_{1}(z), \ldots, \psi_{n}(z)\right)$ be holomorphic self-maps of $\Omega$. The composition operator $C_{\phi}$ induced by $\varphi$ is defined by $\left(C_{\phi} f\right)(z)=f(\phi(z))$ for $z$ in $\Omega$ and $f \in H(\Omega)$.

We recall that the essential norm of a continuous linear operator $T$ is the distance from $T$ to the compact operators, that is, $\|T\|_{e}=\inf \{\|T-K\|: K$ is compact $\}$. Notice that $\|T\|_{e}=0$ if and only if $T$ is compact, so estimates on $\|T\|_{e}$ lead to conditions for $T$ to be compact.

In the past few years, many authors have been interested in studying the mapping properties of the difference of two composition operators, that is, an operator of the form

$$
T=C_{\varphi}-C_{\psi}
$$

The primary motivation for this has been the desire to understand the topological structure of the whole set of composition operators acting on a given function.

The second author was supported in part by the National Natural Science Foundation of China (Grant Nos. 10671141, 10371091).

(C) 2009 Australian Mathematical Society 0004-9727/2009 \$16.00 
Most papers in this area have focused on the classic reflexive spaces, but some classical nonreflexive spaces in the unit disc in the complex plane have also recently been discussed. Hosokawa and Ohno [2,3] gave a characterization of compact difference on Bloch space in the unit disc. Toews [6] and Gorkin and MacCluer [1] independently extended the results to $H^{\infty}\left(B_{n}\right)$ spaces, describing compact difference by Carathéodory pseudo-distance on the unit ball $B_{n}$, which is a generalization of Poinaré distance on the disc.

The present paper continues this line of research, giving some simple estimates of the essential norm for the difference of composition operators induced by $\varphi$ and $\psi$ acting from Bloch space to bounded function space in the unit polydisc $U^{n}$, where $\varphi(z)$ and $\psi(z)$ are two holomorphic self-maps of the unit polydisc in $n$-dimensional complex space. By way of application, a characterization of compact difference is given.

\section{Notation and background}

Throughout this paper, let $D$ be the unit disc in the complex plane $\mathbb{C}, U^{n}$ the unit polydisc in the $n$-dimensional complex space $\mathbb{C}^{n}$, and $\|z\| \|=\max _{j}\left\{\left|z_{j}\right|\right\}$ stands for the supremum norm on $U^{n}$. For a holomorphic function $f$ in $H\left(U^{n}\right)$, define $\nabla f(z)=$ $\left(\left(\partial f / \partial z_{1}\right)(z), \ldots,\left(\partial f / \partial z_{n}\right)(z)\right), R f(z)=\langle\nabla f(z), \bar{z}\rangle, \nabla f(z) u=\langle\nabla f(z), u\rangle$, and $\langle\cdot, \cdot\rangle$ denotes the inner product. For $z, w \in D$, the pseudo-hyperbolic distance between $z$ and $w$ is defined by $\rho(z, w)=|(z-w) /(1-z \bar{w})|$. It is well known that if $f: D \rightarrow D$ is holomorphic, then $\rho(f(z), f(w)) \leq \rho(z, w)$ for any $z, w \in D$. The Bergman metric on the unit polydisc is given by

$$
H_{z}(u, v)=\sum_{j=1}^{n} u_{j} \overline{v_{j}} /\left(1-\left|z_{j}\right|^{2}\right)^{2} .
$$

The Kobayashi distance $k_{U^{n}}$ of $U^{n}$ is given by

$$
k_{U^{n}}(z, w)=\frac{1}{2} \log \frac{1+\left\|\phi_{z}(w)\right\| \mid}{1-\left\|\phi_{z}(w)\right\|},
$$

where $\phi_{z}: U^{n} \rightarrow U^{n}$ is the automorphism of $U^{n}$ given by

$$
\phi_{z}(w)=\left(\frac{w_{1}-z_{1}}{1-\overline{z_{1}} w_{1}}, \ldots, \frac{w_{n}-z_{n}}{1-\overline{z_{n}} w_{n}}\right) .
$$

Let $H^{\infty}$ denote the space of bounded holomorphic functions $f$ on the unit polydisc with the supremum norm $\|f\|_{\infty}=\sup _{z \in U^{n}}|f(z)|$.

According to $[4,5]$, the Bloch space $\mathcal{B}$ in $U^{n}$ consists of those holomorphic functions such that $\|f\|_{\mathcal{B}}=\sup _{z \in U^{n}} Q_{f}(z)<\infty$, where

$$
Q_{f}(z)=\sup \left\{\frac{|\nabla f(z) u|}{H_{z}^{1 / 2}(u, u)}: u \in \mathbb{C}^{n}-\{0\}\right\} .
$$


It is well known that $\|f \circ \phi\|_{\mathcal{B}}=\|f\|_{\mathcal{B}}$ for any automorphism $\phi$ of $U^{n}$, and $\mathcal{B}$ is a Banach space under the norm $\|f\|_{1}=|f(0)|+\|f\|_{\mathcal{B}}$. If we put

$$
G_{f}(z)=\sum_{j=1}^{n}\left(1-\left|z_{j}\right|^{2}\right)\left|\left(\partial f / \partial z_{j}\right)(z)\right|
$$

and $\|f\|=|f(0)|+\sup _{z \in U^{n}} G_{f}(z)$, then it follows from (3.8) and (3.9) in [4] that

$$
\frac{1}{n} G_{f}(z) \leq \max _{1 \leq j \leq n}\left(1-\left|z_{j}\right|^{2}\right)\left|\frac{\partial f}{\partial z_{j}}(z)\right| \leq Q_{f}(z) \leq G_{f}(z) .
$$

This implies that $(1 / n)\|f\| \leq\|f\|_{1} \leq\|f\|$, so $\mathcal{B}$ is also a Banach space with the norm $\|\cdot\|$.

Lemma 1. Assume that $f \in \mathcal{B}$; then

$$
|f(z)-f(w)| \leq n\|f\| \cdot k_{U^{n}}(z, w)
$$

for any $z, w \in U^{n}$.

PROOF.

$$
\begin{aligned}
|f(z)-f(0)| & =\left|\int_{0}^{1} \frac{R f(t z)}{t} d t\right|=\left|\sum_{j=1}^{n} \int_{0}^{1} z_{j} \frac{\partial f}{\partial \zeta_{j}}(t z) d t\right| \\
& \leq \sum_{j=1}^{n} \int_{0}^{1} \frac{\left|z_{j}\right|}{1-\left|t z_{j}\right|^{2}} \frac{\partial f}{\partial \zeta_{j}}(t z) \mid\left(1-\left|t z_{j}\right|^{2}\right) d t \\
& \leq\|f\|_{\mathcal{B}} \sum_{j=1}^{n} \int_{0}^{\left|z_{j}\right|} \frac{1}{1-t^{2}} d t=\frac{1}{2}\|f\|_{\mathcal{B}} \sum_{j=1}^{n} \log \frac{1+\left|z_{j}\right|}{1-\left|z_{j}\right|} \\
& \leq n\|f\|_{\mathcal{B}} \frac{1}{2} \log \frac{1+\|z\| \mid}{1-\|z\|} .
\end{aligned}
$$

The last inequality follows by the fact the map $t \rightarrow \log ((1+t) /(1-t))$ is strictly increasing on $[0,1)$. Setting $z=\phi_{w}(z)$, it follows that

$$
\left.\mid f \circ \phi_{w}(z)\right)-f \circ \phi_{w}(w) \mid \leq n\left\|f \circ \phi_{w}\right\|_{\mathcal{B}} \frac{1}{2} \log \frac{1+\left\|\phi_{w}(z)\right\| \|}{1-\left\|\phi_{w}(z)\right\| \mid} .
$$

Replacing $f \circ \phi_{w}$ by $f \circ \phi_{w} \circ \phi_{w}^{-1}$,

$$
|f(z)-f(w)| \leq n\|f\|_{\mathcal{B}} \frac{1}{2} \log \frac{1+\left\|\phi_{w}(z)\right\| \|}{1-\left\|\mid \phi_{w}(z)\right\|} \leq n\|f\| \cdot k_{U^{n}}(z, w) .
$$

This completes the proof of the lemma. 
Lemma 2. Suppose that $f \in \mathcal{B}$ and, for fixed $0<\delta<1$, let $G=\left\{z \in U^{n}:\|z\| \mid \leq \delta\right\}$. Then

$$
\lim _{r \rightarrow 1} \sup _{\|f\| \leq 1} \sup _{z \in G}|f(z)-f(r z)|=0
$$

PROOF.

$$
\begin{aligned}
\sup _{z \in G}|f(z)-f(r z)| & \\
= & \sup _{z \in G} \mid \sum_{j=1}^{n}\left(f\left(r z_{1}, r z_{2}, \ldots, r z_{j-1}, z_{j}, \ldots, z_{n}\right)\right. \\
& \left.\quad-f\left(r z_{1}, r z_{2}, \ldots, r z_{j}, z_{j+1}, \ldots, z_{n}\right)\right) \mid \\
\leq & \sup _{z \in G} \sum_{j=1}^{n}\left|\int_{r}^{1} z_{j} \frac{\partial f}{\partial z_{j}}\left(r z_{1}, r z_{j-1}, t z_{j}, z_{j+1}, \ldots, z^{n}\right) d t\right| \\
\leq & (1-r) n\|f\| \sup _{z \in G} \frac{1}{1-\|z\|^{2}} \leq \frac{(1-r) n\|f\|}{1-\delta^{2}} .
\end{aligned}
$$

The lemma follows as $r \rightarrow 1$.

\section{Main theorem}

Theorem. For $\delta>0$, write $F_{\delta}=\left\{z \in U^{n}: \max (\|\varphi(z)\|\|,\| \psi(z)\|\|) \leq 1-\delta\right\}$. Suppose that $\varphi, \psi: U^{n} \rightarrow U^{n}$ and $C_{\varphi}-C_{\psi}: \mathcal{B} \rightarrow H^{\infty}$ is bounded. Then

$$
\frac{1}{4} \lim _{\delta \rightarrow 0} \sup _{z \in E_{\delta}}\left\|\phi_{\varphi(z)}(\psi(z))\right\| \mid \leq\left\|C_{\varphi}-C_{\psi}\right\|_{e} \leq 2 n \lim _{\delta \rightarrow 0} \sup _{z \in E_{\delta}} k_{U^{n}}(\varphi(z), \psi(z))
$$

where $E_{\delta}=U^{n}-F_{\delta}$.

PROOF. We consider the upper estimate first. For fixed $0<r<1$, it easy to check that both $C_{r \varphi}$ and $C_{r \psi}$ are compact operators. For any $0<\delta<1$,

$$
\begin{aligned}
\left\|C_{\varphi}-C_{\psi}\right\|_{e} \leq & \left\|C_{\varphi}-C_{\psi}-C_{r \varphi}+C_{r \psi}\right\| \\
= & \sup _{\|f\| \leq 1}\left\|\left(C_{\varphi}-C_{\psi}-C_{r \varphi}+C_{r \psi}\right) f\right\|_{\infty} \\
\leq & \sup _{\|f\| \leq 1} \sup _{z \in F_{\delta}}|f(\varphi(z))-f(r \varphi(z))+f(r \psi(z))-f(\psi(z))| \\
& \quad+\sup _{\|f\| \leq 1} \sup _{z \in E_{\delta}}|f(\varphi(z))-f(\psi(z))-f(r \varphi(z))+f(r \psi(z))| .
\end{aligned}
$$

From Lemma 2, we can choose $r$ sufficiently close to 1 such that the first term of the right-hand side is less than any given $\varepsilon$, and denote the second term by $I$. Using 
Lemma 1, it follows that

$$
\begin{aligned}
I & \leq \sup _{\|f\| \leq 1} \sup _{z \in E_{\delta}}(|f(\varphi(z))-f(\psi(z))|+|f(r \varphi(z))-f(r \psi(z))|) \\
& \leq n \sup _{\|f\| \leq 1} \sup _{z \in E_{\delta}}\left(k_{U^{n}}(\varphi(z), \psi(z))+k_{U^{n}}(r \varphi(z), r \psi(z))\right) \\
& \leq 2 n \sup _{z \in E_{\delta}} k_{U^{n}}(\varphi(z), \psi(z)),
\end{aligned}
$$

last inequality obtaining by $k_{U^{n}}(r \varphi(z), r \psi(z)) \leq k_{U^{n}}(\varphi(z), \psi(z))$. First let $r \rightarrow 1$ and then $\delta \rightarrow 0$; the upper estimate follows.

Now we turn to the lower estimate. For $l=1,2, \ldots, n$, set

$$
E_{\delta}^{l}=\left\{z \in U^{n}: \max \left(\left|\varphi_{l}(z)\right|,\left|\psi_{l}(z)\right|\right)>1-\delta\right\} .
$$

It is easy to see that $E_{\delta}=\bigcup_{l=1}^{n} E_{\delta}^{l}$. For fixed $l(1 \leq l \leq n)$, define

$$
a_{l}=\lim _{\delta \rightarrow 0} \sup _{z \in E_{\delta}^{l}}\left|\frac{\varphi_{l}(z)-\psi_{l}(z)}{1-\overline{\varphi_{l}(z)} \psi_{l}(z)}\right| .
$$

If we put $\delta_{m}=1 / m$, then $\delta_{m} \rightarrow 0$ as $m \rightarrow \infty$.

For the case $\left\|\varphi_{l}\right\|_{\infty}=1$ or $\left\|\psi_{l}\right\|_{\infty}=1$, for large enough $m$ with $E_{\delta_{m}}^{l} \neq \emptyset$, there exists $z^{m} \in E_{\delta_{m}}^{l}$ such that

$$
\lim _{m \rightarrow \infty}\left|\frac{\varphi_{l}\left(z^{m}\right)-\psi_{l}\left(z^{m}\right)}{1-\overline{\varphi_{l}\left(z^{m}\right)} \psi_{l}\left(z^{m}\right)}\right|=a_{l} .
$$

Since $z^{m} \in E_{\delta_{m}}^{l}$ implies that $\left|\varphi_{l}\left(z^{m}\right)\right|>1-\delta_{m}$ or $\left|\psi_{l}\left(z^{m}\right)\right|>1-\delta_{m}$, without loss of generality we assume that $\left|\varphi_{l}\left(z^{m}\right)\right| \rightarrow 1$. Set

$$
f_{m}(z)=\frac{1-\left|\varphi_{l}\left(z^{m}\right)\right|}{1-\overline{\varphi_{l}\left(z^{m}\right)} z_{l}} .
$$

A little calculation shows that $\left\{f_{m}\right\}$ converges to zero uniformly on compact subsets of $U^{n}$ as $m \rightarrow \infty$ and $\left\|f_{m}\right\| \leq 2$ for any $m=1,2, \ldots$ So the compactness of $K$ implies that $\left\|K f_{m}\right\| \rightarrow 0$ whenever $m \rightarrow \infty$, and it follows that

$$
\begin{aligned}
\left\|C_{\varphi}-C_{\psi}-K\right\| & \geq \frac{1}{2} \limsup _{m \rightarrow \infty}\left\|\left(C_{\varphi}-C_{\psi}-K\right) f_{m}\right\|_{\infty} \\
& \geq \frac{1}{2} \limsup _{m \rightarrow \infty}\left(\left\|\left(C_{\varphi}-C_{\psi}\right) f_{m}\right\|_{\infty}-\left\|K f_{m}\right\|_{\infty}\right) \\
& =\frac{1}{2} \limsup _{m \rightarrow \infty}\left\|\left(C_{\varphi}-C_{\psi}\right) f_{m}\right\|_{\infty} \\
& =\frac{1}{4} \limsup _{m \rightarrow \infty}\left|\frac{\varphi_{l}\left(z^{m}\right)-\psi_{l}\left(z^{m}\right)}{1-\overline{\varphi_{l}\left(z^{m}\right)} \psi_{l}\left(z^{m}\right)}\right| \\
& =\frac{1}{4} a_{l}=\frac{1}{4} \lim _{\delta \rightarrow 0} \sup _{z \in E_{\delta}^{l}}\left|\frac{\varphi_{l}(z)-\psi_{l}(z)}{1-\overline{\varphi_{l}(z)} \psi_{l}(z)}\right| .
\end{aligned}
$$


If both $\left\|\varphi_{l}\right\|_{\infty}<1$ and $\left\|\psi_{l}\right\|_{\infty}<1$, in this condition, when $\delta$ is small enough, $E_{\delta}^{l}$ is empty, and without loss of generality we may assume that

$$
\lim _{\delta \rightarrow 0} \sup _{z \in E_{\delta}^{l}}\left|\frac{\varphi_{l}(z)-\psi_{l}(z)}{1-\overline{\varphi_{l}(z)} \psi_{l}(z)}\right|=0 .
$$

Since the above inequality holds for every $1 \leq l \leq n$,

$$
\left\|C_{\varphi}-C_{\psi}\right\|_{e} \geq \frac{1}{4} \max _{1 \leq l \leq n} \lim _{\delta \rightarrow 0} \sup _{z \in E_{\delta}^{l}}\left|\frac{\varphi_{l}(z)-\psi_{l}(z)}{1-\overline{\varphi_{l}(z)} \psi_{l}(z)}\right| .
$$

Now for each $l=1,2, \ldots, n$, we define

$$
b_{l}=\lim _{\delta \rightarrow 0} \sup _{z \in E_{\delta}}\left|\frac{\varphi_{l}(z)-\psi_{l}(z)}{1-\overline{\varphi_{l}(z)} \psi_{l}(z)}\right| .
$$

For any $\varepsilon>0$, there exists a $\delta_{0}$ with $0<\delta_{0}<1$ such that

$$
\left|\frac{\varphi_{l}(z)-\psi_{l}(z)}{1-\overline{\varphi_{l}(z)} \psi_{l}(z)}\right|>b_{l}-\varepsilon
$$

whenever $z \in E_{\delta_{0}}$ and $l=1,2, \ldots, n$. Since $z \in E_{\delta_{0}}^{l}$ implies that $z \in E_{\delta_{0}}$, then by the argument above

$$
\left\|C_{\varphi}-C_{\psi}\right\|_{e} \geq \frac{1}{4} \max _{1 \leq l \leq n}\left(b_{l}-\varepsilon\right)=\frac{1}{4} \lim _{\delta \rightarrow 0} \sup _{z \in E_{\delta}}\left\|\phi_{\varphi(z)}(\psi(z))\right\|-\frac{\varepsilon}{4} .
$$

Now the conclusion follows by letting $\varepsilon \rightarrow 0$.

Corollary. Suppose $C_{\varphi}-C_{\psi}: \mathcal{B} \rightarrow H^{\infty}$ is bounded; then $C_{\varphi}-C_{\psi}$ is compact if and only if

$$
\lim _{\delta \rightarrow 0} \sup _{z \in E_{\delta}}\left\|\phi_{\varphi(z)}(\psi(z))\right\| \|=0 .
$$

Proof. The necessity is obvious by the main theorem. Since $\log ((1+t) /(1-t))$ is strictly increasing on $[0,1)$,

$$
\lim _{\delta \rightarrow 0} \sup _{z \in E_{\delta}}\left\|\phi_{\varphi(z)}(\psi(z))\right\| \|=0
$$

implies that

$$
\lim _{\delta \rightarrow 0} \sup _{z \in E_{\delta}} k_{U^{n}}(\varphi(z), \psi(z))=0,
$$

it follows from the main theorem that $\left\|C_{\varphi}-C_{\psi}\right\|_{e}=0$, so $C_{\varphi}-C_{\psi}$ is compact, and the proof of this corollary is complete. 


\section{References}

[1] P. Gorkin and B. D. MacCluer, 'Essential norms of composition operators', Integral Equations Operator Theory 48 (2004), 27-40.

[2] T. Hosokawa and S. Ohno, 'Topologicial structures of the set of composition operators on the Bloch space', J. Math. Anal. Appl. 34 (2006), 736-748.

[3] 'Differences of composition operators on the Bloch space', J. Operator. Theory 57 (2007), 229-242.

[4] R. Timoney, 'Bloch functions in several complex variables, I', Bull. London Math. Soc. 12(37) (1980), 241-267.

[5] _ _ 'Bloch functions in several complex variables, II', J. Reine Angew. Math. 319 (1980), 1-22.

[6] C. Toews, 'Topological components of the set of composition operators on $H^{\infty}\left(B_{N}\right)$ ', Integral Equations Operator Theory 48 (2004), 265-280.

ZHONG-SHAN FANG, Department of Mathematics, Tianjin Polytechnic University, Tianjin 300160, PR China

e-mail: fangzhongshan@yahoo.com.cn

ZE-HUA ZHOU, Department of Mathematics, Tianjin University,

Tianjin 300072, PR China

e-mail: zehuazhou2003@yahoo.com.cn 